\title{
Desconocimiento de obligaciones tributarias y su incidencia en el Impuesto a la Renta
}

\section{Ignorance of tax obligations and their impact on Income Tax}

\section{Ignorância de obrigações tributárias e seu impacto no Imposto de Renda}

Cruz V. Ponce-Alvarez ${ }^{\mathrm{I}}$

cruzveronica.ponce@unesum.edu.ec

Antonio E. Pinargote-Vásquez II

antonio.pinargote@unesum.edu.ec

Gloria P. Chiquito-Tigua III

g_chiquito@hotmail.com

Erick R. Baque -Sánchez IV

erickbaque@hotmail.com
Mónica del Pilar Quiñonez-Cercado V

mquinonez78@hotmail.com

Gina X. Campozano-Chiquito VI ximecampochi@gmail.com

María G. Salazar-Pilay VII mageosapi@hotmail.com

Recibido: 27 de abril de 2018 * Corregido: 18 de marzo de 2018 * Aceptado: 27 de junio de 2018

I. Docente de la Universidad Estatal del Sur de Manabí, Jipijapa, Ecuador.

II. Docente de la Universidad Estatal del Sur de Manabí, Jipijapa, Ecuador.

III. Master en Contabilidad y Auditoría, Docente de la Universidad Estatal del Sur de Manabí, Jipijapa, Ecuador.

IV. Magister en Contabilidad y Auditoría, Docente de la Carrera de Contabilidad y Auditoría de la Universidad Estatal del Sur de Manabí, Jipijapa, Ecuador.

v. Maestría en Contabilidad y Auditoría, Docente de la Universidad Estatal del Sur de Manabí, Jipijapa, Ecuador.

VI. Docente de la Universidad Estatal del Sur de Manabí, Jipijapa, Ecuador.

VII. Economista; Maestría en Gestión de Proyectos Socio Productivos; Cursando el Doctorado en Ciencias Agrarias; Línea de Investigación Problemas Sociales y Económicos en Agricultura; Docente de la Universidad Estatal del Sur de Manabí, Jipijapa, Ecuador. 


\section{Resumen}

Los ingresos tributarios generan una mayor estabilidad económica y social por lo cual son parámetros de la calidad de vida del ciudadano. Dentro de estos se encuentra el impuesto a la renta el cual toman un porcentaje de los ingresos netos obtenidos por los contribuyentes dependiendo de factores sociales, culturales, geográficos y económicos. Las políticas tributarias del estado deben incentivar a los ciudadanos a declarar estos impuestos y con esto generar una cultura tributaria en la sociedad. Al existir derechos también hay deberes y el no cumplimiento de estos ocasionan sanciones administrativas. Esta investigación tiene como objetivo analizar la incidencia que tiene el desconocimiento de obligaciones tributarias sobre el impuesto a la renta. La metodología utilizada se basó en definir la importancia de los impuestos, conocer los deberes y derechos de las personas naturales y jurídicas, entender las obligaciones tributarias y cómo influyen en el comportamiento socioeconómico del país y por último conocer las sanciones por el no cumplimiento de la declaración de este impuesto. Como conclusión se determinó que el desconocimiento de obligaciones tributarias es función directa de la educación del individuo y de las políticas educativas del estado y por ende afectan, proporcionalmente, al crecimiento del país y la calidad de vida de los ciudadanos.

Palabras Clave: Tributos, impuestos, obligaciones, sanciones y cultura tributaria. 


\begin{abstract}
Tax revenues generate greater economic and social stability, which is why they are parameters of the citizen's quality of life. Within these is the income tax which takes a percentage of the net income obtained by taxpayers depending on social, cultural, geographical and economic factors. The tax policies of the state must encourage citizens to declare these taxes and thereby generate a tax culture in society. When there are rights there are also duties and non-compliance with these causes administrative sanctions. The objective of this research is to analyze the incidence of ignorance of tax obligations on income tax. The methodology used was based on defining the importance of taxes, knowing the duties and rights of natural and legal persons, understanding tax obligations and how they influence the socioeconomic behavior of the country and finally knowing the penalties for non-compliance with the declaration of this tax. As a conclusion, it was determined that the ignorance of tax obligations is a direct function of the education of the individual and of the educational policies of the state and therefore they affect, proportionally, the growth of the country and the quality of life of the citizens.
\end{abstract}

Keywords: Taxes, taxes, obligations, penalties and tax culture. 


\section{Introducción.}

En la actualidad la Administración Tributaria tiene como objetivo fomentar el cumplimiento voluntario de los contribuyentes conforme a la Ley, reduciendo la brecha de incumplimiento, lo que a su vez permite incrementar la eficiencia administrativa de la administración. Este propósito se alinea principalmente con el sistema de impuesto a la renta, ya que éste representa la principal fuente de ingreso gubernamental e imponen un conjunto de obligaciones para la mayoría de contribuyentes.

El sistema tributario exige que todos los contribuyentes tomen conciencia de las obligaciones tributarias, completen y declaren anualmente su impuesto a la renta y así evitar las multas e intereses que se generan por el no cumplimiento establecido en los plazos para declarar y pagar.

Las infracciones de los contribuyentes tendrían para efecto de su juzgamiento y sanción, las infracciones tributarias recaerían en delito, contravenciones y faltas reglamentarias.

A las contravenciones establecidas en el Código y en las demás leyes tributarias se aplicará como pena pecuniaria una multa que no sea inferior a 30 dólares ni exceda de 1.500 dólares de los Estados Unidos de América, sin perjuicio de las demás sanciones, que para cada infracción, se establezcan en las respectivas normas

Los tributos son las presentaciones comúnmente en dinero que el estado exige en ejercicio de su poder de imperio sobre la base de la capacidad contributiva, en virtud de una ley y para cubrir los gastos; que demanda el cumplimiento de sus fines (Quintero, 2015). Dentro de estos tributos están los impuestos, tasas y las contribuciones especiales.

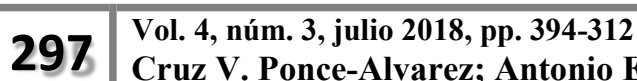


Ahora, existen dos impuestos importantes para la recaudación fiscal. Uno es el impuesto al valor agregado y el otro es el impuesto a la renta. Este último se caracteriza por gravar la totalidad de las rentas obtenidas por las personas, cualquiera sea su procedencia o destino sin distinción alguna, atendiendo a la situación personal del sujeto (cargas de familia, etc.) con tasas progresivas que hagan más fuerte la carga relativa a medida que mayor sea la renta total (García, 1978).

La ley orgánica de régimen tributario interno establece que para efectos de impuesto se considera renta (LEY ORGÁNICA DE RÉGIMEN TRIBUTARIO INTERNO, 2016):

1. Los ingresos de fuente ecuatoriana obtenidos a título gratuito o a título oneroso provenientes del trabajo, del capital o de ambas fuentes, consistentes en dinero, especies o servicios; $y$

2. Los ingresos obtenidos en el exterior por personas naturales domiciliadas en el país o por sociedades nacionales, de conformidad con lo dispuesto en el artículo 98 de esta Ley.

El servicio de rentas internas (SRI) también establece que las personas naturales deben presentar una declaración anual de Impuesto a la Renta, cuando sus ingresos brutos del ejercicio anterior superen la fracción básica establecida en la Tabla 1 del impuesto que se fija para cada año (S.R.I., 2018). 
Tabla 1.- Fracción básica establecida de los últimos 4 años para el pago del impuesto a la renta

\begin{tabular}{|c|c|}
\hline Año & Fracción básica \\
\hline 2015 & $\$ 10.800,00$ \\
\hline 2016 & $\$ 11.170,00$ \\
\hline 2017 & $\$ 11.290,00$ \\
\hline 2018 & $\$ 11.270,00$ \\
\hline
\end{tabular}

Ahora, una de las ventajas del impuesto a la renta, frente al IVA, es que tiene variadas estructuras de tipos y bases, yendo desde los más complejos modelos integrales hasta los más simples de tasa uniforme, y exhibe similar diversidad de exenciones e incentivos (Barreix y Roca, 2007). En cambio, el impuesto al valor agregado permite no gravar la inversión ni exportar impuestos o disimular subsidios.

Por lo cual, es un deber y derecho el pago de los impuestos, sean personas naturales o jurídicas, ya que el estado invierte en las instituciones públicas como educación, salud, seguridad, agua, luz, aseo urbano, entre otros. Es por ello que es necesario tener la obligación moral de contribuir al financiamiento de estas organizaciones las cuales garantizan una mejor calidad de vida. El desconocimiento de la declaración de este impuesto no lo exime de responsabilidades administrativas y penales ya que escapa de los deberes formales del sistema de rentas internas el cual establece que se deben presentar todos los documentos, declaraciones, informe y libros pertinentes a las obligaciones tributarias. 
Por lo tanto, aquellos individuos que generan una renta lucrativa deben contribuir con el pago tributario, amparado por la ley y la constitución de la república del Ecuador, indistintamente si la falta de pago es voluntaria o fuera de las condiciones explicitadas normativamente, entra en escena el régimen sancionatorio tributario, como instrumento disuasivo para promover el pago del tributo, contentivo de dos propósitos, uno de orden preventivo y otro de carácter represivo. (Mogrovejo, 2011).

\section{Método.}

El desarrollo de esta investigación se basó en un procedimiento metodológico basado en la importancia del impuesto a la renta, los deberes y derechos de los contribuyentes, las obligaciones tributarias y las sanciones por la no declaración del impuesto. La metodología se basó en técnicas basadas en revisión de textos, documentos y artículos científicos publicados disponibles en la web. Aunado a esto también se aplicaron entrevistas distintas personas de diferentes estratos sociales y de diferentes edades con el fin de conocer cuáles son los factores que pueden impulsar el desarrollo tributario en nuestro país.

\section{Resultados.}

Importancia de los impuestos

Los tributos han existido desde la misma evolución del hombre, pero han tomado mayor énfasis cuando eran tomados como único financiamiento para los enfrentamientos bélicos entre las naciones, sobre todo de países desarrollados como Europa y Estados Unidos. La única manera de

\footnotetext{
300 Vol. 4, núm. 3, julio 2018, pp. 394-312 
costear los gastos producidos por las guerras era a través de figuras tributarias que captaban aspectos especiales de ciertas rentas de algunos capitales provenientes de la sociedad.

En América Latina, la repercusión de este movimiento tributario se produce con algún retraso, pudiendo indicarse que entre los años 1920 y 1935 se asiste a la difusión de este impuesto, quedando incorporado desde entonces a los sistemas tributarios, en los cuales tiene un peso que varía de país en país, pero en general puede considerarse de gran trascendencia (García, 1978).

Por lo cual es importante entonces poder definir el concepto de retenciones sobre esas rentas y es allí donde entran los tributos como los medios económicos de los que se sirven el estado y algunas instituciones pare poder solventar sus obligaciones. De igual manera es importante resaltar que los impuestos tributarios son ingresos que percibe el estado por la prestación de servicios públicos y concesión de permisos y licencias; así como de rentas que derivan de la actividad empresarial y de su propio patrimonio. También comprende los recursos por multas e infracciones varias y no especificadas (Quintero, 2015).

Conociendo estos términos entonces se procede a resaltar los diferentes tipos de tributos los cuales van a fortalecer la economía del estado. Estos tipos se clasifican en impuestos, tasas y contribuciones especiales. El impuesto es el tributo más importante y significativo para la retención de dinero proveniente de algún capital. Pero para que exista una efectiva contribución debe existir una efectiva relación entre el estado y los ciudadanos, es decir, los ingresos tributarios dependen si y solo si de la relación entre el estado y los individuos que la conforman.

La relación Estado-ciudadano va más allá de un simple contrato de contraprestaciones; se concreta en lo fiscal, como canal para alcanzar la justicia distributiva en la que los ciudadanos

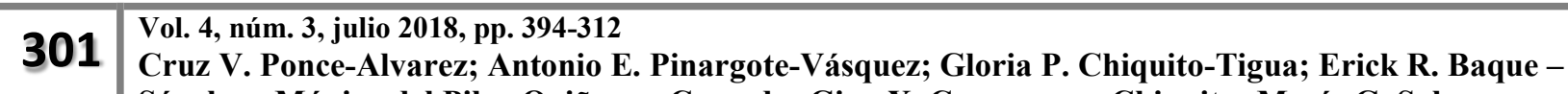
Sánchez; Mónica del Pilar Quiñonez-Cercado; Gina X. Campozano-Chiquito; María G. SalazarPilay
} 
perciben el pago de impuestos de acuerdo a sus valores morales, como una contribución al bien común y actúan honestamente al aportar su contraparte, incluso si no reciben los bienes y servicios esperados por su contribución (Bedoya y Vásconez, 2011).

Por lo cual esta relación tiende a ser una relación dar-recibir a simple vista lo cual genera que muchos de los individuos no estén obligados a cumplirla. Aquí entra en juego las obligaciones que tiene la sociedad para con el pago tributario, es decir las obligaciones tributarias. Estas Obligación Tributaria es definida como el vínculo jurídico personal, existente entre el Estado o las entidades acreedoras de tributos y los contribuyentes o responsables de aquellos, en virtud del cual debe satisfacerse una prestación en dinero, especies o servicios apreciables en dinero, al verificarse el hecho generador previsto por la ley (Sarmiento, 2007)

\section{Deberes y derechos de los contribuyentes}

Pero si existe una obligación también debe existir un derecho para los contribuyentes entonces debe haber beneficios que pueden favorecer la inversión en una economía pequeña y abierta las cuales deben favorecer elementos clave como la seguridad jurídica de los derechos de propiedad, estabilidad tributaria donde exista menores cambios de política tributaria y menor discrecionalidad posible en su aplicación y una tasa efectiva de imposición baja, uniforme y competitiva internacionalmente (Roca, 2010).

Hay derechos para las personas naturales que establecen los desgravámenes para la declaración del impuesto a la renta los cuales son los gastos personales del contribuyente como su carga familiar (padres, conyugue o pareja en unión de hechos e hijos mayores de edad) que no perciban ingresos gravados y dependen de este. Esta deducción no debe de exceder del 50\% total de

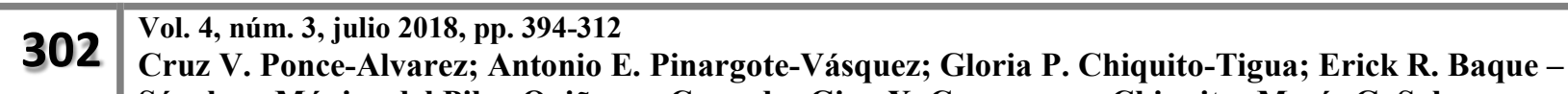
Sánchez; Mónica del Pilar Quiñonez-Cercado; Gina X. Campozano-Chiquito; María G. SalazarPilay
} 
los ingresos gravados del contribuyente o del 1,3 veces mayor a la fracción básica desgravada del impuesto a la renta de personas naturales, exceptuando los gastos de salud en enfermedades crónicas debidamente certificada por la Autoridad Sanitaria Nacional (Servicio de Rentas Internas, 2018).

Los gastos personales que se pueden deducir son concepto de vivienda, alimentación, vestimenta, salud y educación incluyendo en este rubro arte y cultura. Estos gastos se muestran el Tabla 2 (Servicio de Rentas Internas, 2018).

Ahora, el comportamiento del impuesto a la renta va a depender de variables geográficas, políticas, económicas, sociales y culturales de la sociedad. Una marcada diferenciación se puede observar en la Tabla 3 la cual muestra que las mayores diferencias entre estos dos grupos de países se originan en el gravamen a la renta personal y las contribuciones a la seguridad social; en cambio, el IVA y el impuesto a la renta empresarial lucen relativamente similares, pese a las importantes disparidades de desarrollo e ingreso entre ambos grupos (Barreix y Roca, 2007). 


\section{Tabla 2.- Límites para la deducción de gastos personales}

\begin{tabular}{|c|c|c|c|c|c|c|}
\hline \multicolumn{7}{|c|}{ Limites para la deduccion ce gastos personalea } \\
\hline \multirow[t]{2}{*}{ pubro } & Continente & Galtpagos: & \multirow{2}{*}{$\begin{array}{l}2017 \\
\text { Continente } \\
\text { (Fraceion } \\
\text { basica } \\
\text { desgravada } \\
\text { IR USD } \\
11.290 \text { ) }\end{array}$} & $\begin{array}{l}2017 \\
\text { Galspapos }\end{array}$ & $\begin{array}{l}2018 \\
\text { Continente }\end{array}$ & $\begin{array}{l}2018 \\
\text { Galspagos }\end{array}$ \\
\hline & $\begin{array}{l}\text { Nomero de va } \\
\text { fracolon bats } \\
\text { desgravada d } \\
\text { ia Renta de pi } \\
\text { naturales }\end{array}$ & $\begin{array}{l}\text { ces la } \\
\text { Impuesto a } \\
\text { renas. }\end{array}$ & & $\begin{array}{l}\text { (Fracción } \\
\text { basice } \\
\text { desgravads } \\
\text { IR UsD } \\
11.290)\end{array}$ & $\begin{array}{l}\text { (Fracolon } \\
\text { besica } \\
\text { desoravada } \\
\text { ip USD } \\
11.270 \text { ) }\end{array}$ & $\begin{array}{l}\text { (Fraccion } \\
\text { básica } \\
\text { desgravada } \\
\text { IR USP } \\
11.270 \text { ) }\end{array}$ \\
\hline Vivienda & 0,325 & 0,586 & 3.669 .25 & $6.615,94$ & 3.662 .75 & 6.604 .22 \\
\hline $\begin{array}{l}\text { Educación } \\
\text { incluye rubros } \\
\text { de arte y } \\
\text { cultura } *\end{array}$ & 0,325 & 0,586 & 3.669 .25 & 6.615 .94 & 3.662 .75 & 6.604 .22 \\
\hline Alimentacion & 0,325 & 0,586 & $3.669,25$ & 6.615 .94 & $3,662,75$ & $6.604,22$ \\
\hline Vestimenta & 0,325 & 0.586 & 3.669 .25 & $6.615,94$ & $3.662,75$ & $6.604,22$ \\
\hline Salud & 1,3 & 2.344 & $14.677,00$ & $26,463,76$ & $14.651,00$ & $26.416,88$ \\
\hline $\begin{array}{l}\text { Total gastos } \\
\text { personales } \\
\text { (Hasta el sox } \\
\text { de los } \\
\text { ingresos } \\
\text { gravados del } \\
\text { contribuyente } \\
\text { sin que } \\
\text { supere los } \\
\text { valores } \\
\text { seflalados en } \\
\text { esta fila) }\end{array}$ & 1,3 & 2,344 & $14.677,00$ & $26.463,76$ & $14.651,00$ & 26.416 .85 \\
\hline
\end{tabular}

* Para la provincia de Galápagos el limite se obtiene al multiplicar el monto máximo para cada rubro en el continente por el Índice de Precios al Consumidor Espacial de Galápagos 1,803 establecido desde el ejercicio físcal 2016

** Los rubros de gastos de arte y cultura se incorporaron como dechucibles desde el ejercicio fiscal 2017 (su limite de deducibilidad en este ejercicio fue de 1,3 veces la fracción), y desde el ejercicio fiscal 2018 estos rubros se incluyeron en el de educación, compartiendo el limite de 0,325 veces.

**8 En estos casos tanto los gastos por rubro de salud como el total de gastos personales pueden ser deducibles hasta 2 fracciones básicas desgravadas de Impuesto a la Renta, debiendo respetarse los límites individuales para el resto de rubros. Ejemplo: si durante el ejercicio 2018 el contribuyente obtiene USD 30.000 como ingresos gravados y él o sus dependientes tienen gastos por este tipo de enfermedades, sus gastos deducibles por salud podrán ser de hasta USD 22.540 . Si el contribuyente tuviese este tipo de gastos sustentados hasta USD 14.000 podria completar el limite antes indicado con el resto de rubros de gastos personales, respetando los limites individuales previstos para cada uno de ellos. 
El impuesto a la renta, como tributo basado en la capacidad de pago, es el eje principal de cualquier sistema tributario que busca una mejor redistribución de los ingresos (Ramírez, Cano y Oliva, 2009). Y está basado en el concepto de renta la cual radica en que la vida económica genera satisfacciones o enriquecimiento de muy distinta índole por lo cual algunos de estos enriquecimientos resultan gravados y otros no (García, 1978).

Tabla 3.- Organización de Cooperación y Desarrollo Económicos (OCDE) y América Latina: pilares tributarios, 2004 (Porcentajes del producto interno bruto)

\begin{tabular}{crc}
\hline & OCDE & América Latina $^{\mathrm{a}}$ \\
\hline Ingreso tributario $^{\mathrm{b}}$ & 35,9 & 20,2 \\
Impuesto al valor agregado (IVA) $^{\mathrm{c}}$ & 6,7 & 5,8 \\
Impuesto a la renta & 12,5 & 3,8 \\
A la renta empresarial & 3,4 & 2,6 \\
A la renta personal & 9,1 & 1,2 \\
Seguridad social $^{\mathrm{d}}$ & 9,3 & 2,8 \\
\hline
\end{tabular}

Fuente: Organización de Cooperación y Desarrollo Económicos (OCDE), Comisión Económica para América Latina y el Caribe (CEPAL), Banco Interamericano de Desarrollo (BID) y Fondo Monetario Internacional (FMI).

a Incluye ingresos por recursos del petróleo en Colombia, Ecuador, México y Rep. Bolivariana de Venezuela; minerales en Chile y Bolivia, e hidroelectricidad en Paraguay.

b Incluye seguridad social (pensiones).

c Incluye el impuesto sobre la circulación de mercaderías y prestación de servicios (ICSM) en Brasil.

d Incluye contribuciones a los sistemas públicos. 


\section{Obligaciones tributarias}

La finalidad de la recaudación provenientes del impuesto a la renta es financiar el presupuesto del Estado, y de esta manera atender las necesidades de carácter general como educación, salud, seguridad, agua, luz, recolección de basura, entre otros. El estado tiene numerosas responsabilidades como la dirección de la economía, la defensa nacional, la vigilancia de las actividades económicas privadas, la promoción de la agricultura, industria, comercio y turismo (Quintero, 2015). El buen funcionamiento de estos servicios públicos hace de que el país exista un desarrollo económico y social con el fin de aumentar la calidad de vida de los ciudadanos.

Por lo cual, las obligaciones tendrán un valor cuantitativo dependiendo si el contribuyentes persona natural o persona jurídica.

Si es persona jurídica entonces se aplica un $22 \%$, sobre su base imponible, los ingresos de sociedades domiciliadas o constituidas en el Ecuador. Si la sociedad tiene socios, partícipes, constituyentes, beneficiarios o similares con un capital social del 50\% tendrá una tarifa impositiva del 25\% (Servicio de Rentas Internas, 2018).

Para liquidar el Impuesto a la Renta en el caso de las personas naturales y de las sucesiones indivisas, se aplicará a la base imponible las siguientes tarifas mostrada en la Tabla 4 provenientes del artículo 36 de la Ley Orgánica de Régimen Tributario Interno (Servicio de Rentas Internas, 2018):

306 Vol. 4, núm. 3, julio 2018, pp. 394-312 
Tabla 4.- Base imponible para personas naturales del año 2017 expresado en dólares

\begin{tabular}{c|c|c|c|}
\hline $\begin{array}{c}\text { Fracción } \\
\text { Básica }\end{array}$ & $\begin{array}{c}\text { Exceso } \\
\text { hasta }\end{array}$ & $\begin{array}{c}\text { Impuesto } \\
\text { Fracción Básica }\end{array}$ & $\begin{array}{c}\text { \% Impuesto } \\
\text { Fracción } \\
\text { Excedente }\end{array}$ \\
\hline 0 & 11.290 & - & $0 \%$ \\
\hline 11.290 & 14.390 & - & $5 \%$ \\
\hline 14.390 & 17.990 & 155 & $10 \%$ \\
\hline 17.990 & 21.600 & 515 & $12 \%$ \\
\hline 21.600 & 43.190 & 948 & $35 \%$ \\
\hline 43.190 & 64.770 & 4.187 & $20 \%$ \\
\hline 115.140 & En adelante & 22.534 & $15 \%$ \\
\hline 64.770 & 86.370 & 8.503 & $25 \%$ \\
\hline
\end{tabular}

Ahora, para hacer cumplir este objetivo de crecimiento económico es necesario comprender la necesidad de realizar el pago de los mismos y esto tiende a ser una combinación de probabilidades entre la sanción y la auditoría. Definitivamente está relacionada con la moral tributaria de los ciudadanos que a su vez relaciona con los valores, la percepción social y económica, la reciprocidad, el riesgo, la facilidad de pago y el conocimiento y finalmente, con las características sociodemográficas y socio-económicos (Bedoya y Vásconez, 2011). Por lo cual se construye entonces una variable categórica en la que se identifican tres niveles como se muestra en la Tabla 5. 
Tabla 5.- Distribución para la frecuencia de moral tributaria para los ciudadanos ecuatorianos

\begin{tabular}{|c|c|c|c|c|}
\hline Orden & Categoria Moral Tributaria & Frecuencia & Porcentaje & Acumulado \\
\hline 3 & $\begin{array}{l}\text { EI NO PAGAR impuestos } \\
\text { NO se puede justificar en } \\
\text { ningún caso }\end{array}$ & 2,227 & 53.53 & 53.53 \\
\hline 2 & $\begin{array}{l}\text { En ciertas ocasiones las } \\
\text { personas o empresas } \\
\text { pueden tener justificación } \\
\text { para no pagar impuestos }\end{array}$ & 1,474 & 35.43 & 88.97 \\
\hline 1 & $\begin{array}{l}\text { Es justificable NO PAGAR } \\
\text { impuestos en el Ecuador }\end{array}$ & 179 & 4.3 & 93.27 \\
\hline \multirow[t]{2}{*}{9} & No sabe/No responde & 280 & 6.73 & 100.00 \\
\hline & Total & 4,160 & 100 & \\
\hline
\end{tabular}

Fuente: (Bedoya y Vásconez, 2011)

Esta Tabla 5 refleja claramente el comportamiento de la ciudadanía ecuatoriana con respecto al desconocimiento de las obligaciones tributarias y por ende afecta a cultura tributaria que se debe implementar en el país. Es importante resaltar que el termino cultura tributaria es función de los deberes formales de todo ciudadano, es decir que el individuo tiene la obligación de declarar y pagar los tributos generados por las rentas de un determinado período. Este desconocimiento de la normativa tributaria o altos costos asociados al pago de impuestos, originados muchas veces por leyes complejas, pueden no sólo inclinar la relación ‘costo-beneficio' hacia la evasión, sino además generar resentimientos que debilitarán la conciencia moral de los contribuyentes empujándolos incluso a evadir como una forma de 'castigo' para la Administración Tributaria (Bedoya y Vásconez, 2011). Esta moral tributaria incide directamente en la recaudación del impuesto a al renta.

Para evitar sanciones por la no declaración del impueso a la renta existen plazos que varían de acuerdo al noveno dígito de la cédula o RUC, de acuerdo al tipo de contribuyente. Esto se 
muestra en la Tabla 6 contemplado en el artículo 40 de la LORTI (Servicio de Rentas Internas, 2018):

Tabla 6.- Plazos para la declaración del impuesto variando el noveno dígito de la cedula o RUC

\begin{tabular}{|c|c|c|}
\hline $\begin{array}{l}\text { Noveno dígito } \\
\text { RUC/CÉDULA }\end{array}$ & $\begin{array}{c}\text { Plazo para } \\
\text { Personas } \\
\text { Naturales }\end{array}$ & $\begin{array}{l}\text { Plazo para } \\
\text { Sociedades }\end{array}$ \\
\hline 1 & 10 de marzo & 10 de abril \\
\hline 2 & 12 de marzo & 12 de abril \\
\hline 3 & 14 de marzo & 14 de abril \\
\hline 4 & 16 de marzo & 16 de abril \\
\hline 5 & 18 de marzo & 18 de abril \\
\hline 6 & 20 de marzo & 20 de abril \\
\hline 7 & 22 de marzo & 22 de abril \\
\hline 8 & 24 de marzo & 24 de abril \\
\hline 9 & 26 de marzo & 26 de abril \\
\hline 0 & 28 de marzo & 28 de abril \\
\hline
\end{tabular}

Sanciones tributarias

Ahora indistintamente si existe desconocimiento o no del pago tributario se debe realizar una sanción tributaria la cual es consecuencia de los actos u omisiones que se encuentran configurados como infracción, con fines preventivos y represivos manifestados, en el primer caso, a través de la 
disuasión general para no cometerla, y, en el segundo, con el castigo por habérsela perpetrado (Mogrovejo, 2011).

En el artículo 100 de la LORTI se plantea el cobro de multas a los contribuyentes que no declaren los tributos serán sancionados con el 3\% por cada mes de retraso en la presentación de la declaración, la cual se calculará sobre el impuesto causado según la respectiva declaración. Ahora si el contribuyente no cancela las multas generadas por la no declaración entonces el SRI le aumentará en un $20 \%$. Si sigue las infracciones entonces se procederá a los previsto en el Libro Cuarto del Código Tributario (LEY ORGÁNICA DE RÉGIMEN TRIBUTARIO INTERNO, 2016)

\section{Discusión y conclusiones.}

El impuesto a la renta es uno de los principales tributos de la nación ya que recaudan la mayor cantidad de capital con el cual se podrá disponer de unos mejores servicios y así obtener una calidad de vida óptima. Ecuador plantea los principios para la declaración en su artículo 300 de la Constitución:

El régimen tributario se regirá por los principios de generalidad, progresividad, eficiencia, simplicidad administrativa, irretroactividad, equidad, transparencia y suficiencia recaudatoria. Se priorizarán los impuestos directos y progresivos.

Los contribuyentes están divididos en personas naturales y jurídicas las cuales tienen deberes y derechos que les otorgan las leyes. Para las personas jurídicas existen exoneraciones que permiten aún más la inversión en el desarrollo del país. Estos beneficios tributarios están concebidos a factores como la inversión-reinversión de capital. Si esto sale del circulo entonces debe ser gravado

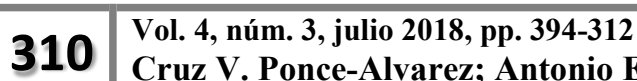


Para las personas naturales corresponden otro tipo de exoneraciones las cuales están determinados por gastos personales, de salud, educación entre otros. No solo para el contribuyente sino también para la carga familiar que depende económicamente de este.

Los contribuyentes deben tener una obligación tributaria constante en el tiempo y esto genera una cultura tributaria en la sociedad. Esta cultura tributaria va a depender de las políticas del Estado para redistribuir estos ingresos en mejorar muchos aspectos de los gastos públicos. Los ingresos tributarios son función del destino de los tributos del país.

Las personas naturales y jurídicas no están exentos de sanciones las cuales rigen el control del buen funcionamiento del sistema tributario nacional. Estas sanciones van desde multas administrativas por la no declaración del impuesto hasta sanciones penales. Estas sanciones dependen de declaración del impuesto y son porcentajes mensuales que irán en aumento sino se cancela dichas multas.

Por lo tanto, el desconocimiento de obligaciones tributarias es función directa de la educación del individuo y de las políticas educativas del estado hacia los ciudadanos ya que no existen programas educativos donde prevalezcan la finalidad del impuesto, la influencia sobre el crecimiento del país a través de la economía y desarrollo social y que la no declaración trae consecuencias administrativas y penales. Por supuesto esta divulgación fiscal permite mejorar la recaudación cada año a fin de eliminar o disminuir la brecha fiscal que permita mayor recaudación para mejores servicios para todos. Sin embargo, el comportamiento del ciudadano siempre será buscar la forma de pagar menos tributos; por lo tanto, la tarea de continuar capacitando, controlando y sancionando por parte de la Administración no puede mermarse sino al contrario incrementarse y

311 Vol. 4, núm. 3, julio 2018, pp. 394-312 
perfeccionarse con la contribución de la tecnología a fin de que su nuevo objetivo sea que los pagos que realicen se ajusten a su realidad económica de contribución y no se trate de cumplimientos a medias por parte de los contribuyentes.

\section{Referencias.}

Alarcón, M. (2009). El impuesto a la renta para el Ecuador: Un sistema distributivo. Fiscalidad, $105-150$.

Barreix, A., \& Roca, J. (2007). Reforzando un pilar fiscal: el impuesto a la renta. Revista de la Cepal, 123-142.

Bedoya, A., \& Vásconez, B. (2011). Entendiendo la Moral Tributaria en Ecuador. 45․ ASAMBLEA GENERAL DEL CIAT (págs. 1-41). Quito - Ecuador: Centro Interamericano de Administraciones Tributarias - CIAT.

García, R. (1978). Impuesto sobre la Renta: Teoría y Técnica del Impuesto. Buenos Aires Argentina: Centro Interamericano de Estudios Tributarios (CIET) - OEA.

Internas, S. d. (24 de Julio de 2018). SRI . Obtenido de http://www.sri.gob.ec/web/guest/impuestorenta

LEY ORGÁNICA DE RÉGIMEN TRIBUTARIO INTERNO (CONGRESO NACIONAL 29 de Abril de 2016).

Mogrovejo, J. C. (2011). Las sanciones en materia tributaria en el Ecuador. Revista de Derecho UASB-Ecuador, 21-42.

Quintero, J. (2015). LOS TRIBUTOS VINCULADOS” "IMPUESTOS, TASAS Y CONTRIBUCIONES ESPECIALES EN ECUADOR. Loja - Ecuador: Tesis de Grado para optar a abogado - Universidad Nacional de Loja.

Ramírez, J., Cano, L., \& Oliva, N. (2009). Impuesto a la Renta de Personas Naturales en Relación de Dependencia Un Análisis de Equidad y Redistribución. Quito - Ecuador: Centro de Estudios Fiscales - Documento de Trabajo No. 2010-01.

Roca, J. (2010). Evaluación de la efectividad y eficiencia de los beneficios tributarios. Washington, D.C., U.S.A.: Banco Interamericano de Desarrollo - Sector de Capacidad Institucional y finanzas.

S.R.I. (2018). Deberes Formales. Yo construyo mi Ecuador , 1-27.

Sarmiento, C. (2007). LA CULTURA TRIBUTARIA EN ECUADOR. Guayaquil - Ecuador: Material didactico de la Universidad de Guayaquil. 